\title{
V. \\ Kritische und experimentelle Untersuchungen über die Irisbewegungen und iiber den Einfluss ron Giften auf dieselben, besonders des Cocains. \\ Von
}

Dr. Ph. Iimbourg, Augenarzt.

\section{Anatomisches über die Dilatatorfrage.}

Neben der Thätigkeit des anatomisch wohl ausgebildeten M. sphineter pupillae ist an der Iris besonders die active Dilatation der Pupille aufgefallen, welche zumal bei Reizung des Halssympathicus erfolgt (Biff $\left.\dot{i}^{1}\right)$. Dieser physiologische Antagonismus schien einen besonderen Muskel als Antagonisten des Sphincters zu verlangen. Dem anatomischen Nachweis boten sich jedoch grosse Schwierigkeiten. Von Vögeln, welche einen quergestreiften Sphincter besitzen ( $\mathrm{Kr}^{\circ} \mathrm{hn}^{2}{ }^{2}$ ), Dogiel ${ }^{3}$ ), Gruenhagen ${ }^{4}$, Faber ${ }^{5}$ ) u. A.), wurde ziemlich übereinstimmend ein ebenfalls quergestreifter Dilatator beschrieben ( $\mathrm{K} \ddot{\mathrm{l}} \mathrm{l}$ liker ${ }^{6}$ ), H. Müller ${ }^{7}$ ), Gruenhagen, v. Hüt tenbrenner ${ }^{8}$ ), Dogiel, Faber, Koganeï ${ }^{9}$ ) u. A.). Wie aber die Untersuchungen

1) Citirt nach J. Budge, Ueber die Bewegung der Iris. Braunschweig 1855. S. 107 u. 91.

2) Archir f. Anat. u. Physiol. 1837. S. 357.

3) Archiv f, mikrosk. Anat. Bd. VI. S. 89. 1870.

4) Virchow's Arch. Bd. XXX. S. 481. 1864. Ferner Berlin. klin. Wochenschr. 1865. Nr. 23 u. 24. - Zeitschr. f. rat. Med. Bd. XXVIII. 1866 u. Bd. XXXI. 1868. (Zusatz zur Arbeit von Schur) u. Bd. XXXVI. 1869. - Archiv f. d. ges. Physiol. Bd. III. S. 440. 1870. - Archiv f. mikrosk, Anat. Bd. IX. 1873. S. 286 u. 726. Archiv f. d. ges. Physiol. Bd. X. S. 165. 1875. - Anatomischer Anzeiger. Bd. III. Nr. 1. 1888.

5) Der Bau der Iris des Menschen und der Wirbelthiere. Leipzig 1876.

6) Zeitschr. f. wissensch. Zoologie. Bd. I. S. 54. 1849. - Handbuch der Gewebelehre des Menschen. 5. Aufl. 1867. S, 667.

7) Archiv f. Ophthalm. Bd. III (1), 1857.

8) Sitzungsber. der Wiener Akad. Bd. LVII (1). S. 515. 1868.

9) Untersuchungen über den Bau der Iris des Menschen und der Wirbel. thiere. Leipzig 1876. 
von $\mathrm{Budge}$ und in neuerer Zeit von $\mathrm{Michel}^{1}$ ) zeigen, sind die Angaben über die Iris der einzelnen Vogelspecies nicht frei von Widersprïchen.

Bei Säugethieren hatte das Bestreben, die anatomische Grundlage für die Anschauung zu schaffen, dass die Existenz eines musculösen Dilatators nicht $z u$ entbehren sei, einen wechselnden Erfolg. Man legte verschiedenen Gebilden jene Rolle bei. Zunächst sollte der Dilatator eine ununterbrochene Membran aus radiär angeordneten glatten Muskelzellen an der hinteren Begrenzung des Iristroma vorstellen (Henle ${ }^{2}$, Merkel ${ }^{3}$ ), Jeropheeff ${ }^{4}$, Jwanoff ${ }^{5}$ ), Faber, We is cher ${ }^{b}$ ) u. A.). Gegen diese Auffassung der hinteren Grenz= lamelle, welche $\mathrm{Bruch}$ und $\mathrm{H}$. M ü ller als mit der elastischen Grundlamelle der Chorioidea im Zusammenhang stehend beschrieben hatten, wandten sich Mayer ${ }^{7}$ ), Hampeln ${ }^{8}$ ) u. A. Am meisten wurde jene Anschaung bekämpft von Gruenhagen, welcher die Membran den elastischen Häuten zuzählte. Nach Michel ist die hintere Begrenzung'sschicht aus Faserzellen zusammengesetzt, welche beim Menschen und Kaninchen den Sphincterelementen ähnlich sind, beim Schaf, Schwein und bei Vögeln dagegen Pigment führen. Schwalbe ${ }^{9}$ ) hält die hintere Grenzschicht für eine elastische Membran von feiner radiärer Streifung, deren Dicke beim Menschen geringer ist, als der Querschnittsdurchmesser glatter Muskelfasern. Die Deutung werde dadurch erschwert, dass ibr in der Regel spindelförmige Zellen vom äusseren Blatt der secundären Augenblase und einzelne der Vorderfläche anliegende glatte Muskelfasern anhaften. Eversbusch ${ }^{10}$ ) schliesst sich dieser Schilderung an.

Man hat ferner radiär verlaufende Muskelfasern im Iristroma für

1) Die histologische Structur des Iristroma. Erlangen 1875. - Ber. über die 13. Versamml. der ophth. Ges. zu Heidelberg. 1881. S. 106. - Archiv f. Ophthalm. Bd. XXVII (2). S. 171.

2) Handb. d. system. Anatomie d. Mensch. 2. Aufl. Bd. II. S. 662. 1873.

3) Zeitschr. f. rat. Medicin. Bd. XXXI. 1868 u. Bd. XXXIV. 1869. - Die Musculatur der menschlichen Iris. Rostock 1873.

4) Citirt nach I wa no fif.

5) Handb. d. ges. Augenheilkunde nach Gräfe und Sämisch. Bd. I. S. 28.

6) Ein Beitrag zur Musculatur d. Iris. Inaug.-Diss. Greifswald 1883.

7) Verhandl. d. naturh. Vereines der prenss. Rheinlande und Westphalens. Bd. X. S. 48.1853.

8) Ein Beitrag zur Anatomie d. Iris. Inaug.-Diss. Dorpat 1869.

9) Lehrbuch d. Anatomie d. Sinnesorgane. Erlangen 1887. S. 202-207.

10) Zeitschr. f. vergl. Augenheilk. 1882. S. 49. - Ber. über d. 16. Versamml. d. ophth. Ges. zu Heidelberg. 1884. S. 54 u. 136. - Zeitschr. f. vergl. Augenheilk. Bd. III. 1885. S. 33 u. 145. 
Kritische u. experiment. Untersuchungen über die Irisbewegungen u. s. w. 95

den Dilatator gehalten (Kölliker, Brï cke11), Dogiel, Eversbusch, Michel, Ewing ${ }^{2}$ ) u. A.). Radiäre glatte Muskelfasern, welche zum Sphincter sich verhalten, wie die Speichen zur Nabe des Rades, sind auch von Gruenhagen beschrieben, jedoch als Insertionsbiundel des Sphincters gedeutet worden. Beim Menschen sind sie nach Schwalbe nur schwach entwickelt, so dass sie den Namen eines M. dilatator pupillae kaum verdienen. D on de rs ${ }^{3}$ ) konnte sich von der Existenz radiärer Muskelfasern, welche unabhängig von den Gefässen vorkommen, nicht überzeugen.

Nach Koganeï feblt beim Menschen und bei den meisten Säugethieren der Dilatator überhaupt; schwach ausgebildet sei er beim Kaninchen, sehr stark dagegen bei der Fischotter. Auf Grund gleichzeitiger Untersuchungen behauptet Eversbusch, dass weder in der einen noch in der anderen Form ein gesonderter Dilatator besteht. Man sei in der Iris leicht Täuschungen ausgesetzt, indem den Gefässen entlang verlaufende Bündel und in der Irisperipherie gelegene Arkadenbruchstiicke, welche für Theile des Dilatators gehalten werden, nervöser Natur sind. Schief getroffene Sphincterfasern täuschten vom Sphincter nach der Peripherie sich abzweigende Bündel vor. Radiäre Muskelfasern lassen sich nach ihm ausschliesslich bei spaltförmigen Pupillen nachweisen. Sie finden sich an den Endpunkten des grössten Durchmessers, und zwar in um so grösserer Zahl, je enger der von der Iris gebildete Spalt sein kann. Im Kaninchenauge kommen sie daher nur spärlich vor, reichlicher bei Katzen. In der dreieckigen Fischotterpupille befinden sich radiäre Muskelfasern allein an den drei Ecken. Wegen dieser auschliesslichen Beziehung der Faserztige zu der Form der Pupille bezeichnet Eversbusch dieselben als accessorische Sphincterinsertionen. Auch Debierre ${ }^{4}$ ) beschreibt nur einen einzigen Maskel in der Iris.

Man ist wohl berechtigt, aus diesen Arbeiten den Schluss zu ziehen, dass ein musculöser Dilatator für kein Säugethier sicher bewiesen ist. Die elastische Natur der Bruch'schen Membran dürfte feststehen. Das Vorkommen selbständiger, nicht zum Sphincter gehörender glatter Musculatur von radiärem Verlauf scheint unerheblich oder zweifelhaft zu sein. Der Standpunkt, welchen Gruen-

1) Anatomische Beschreibung des menschlichen Augapfels 1847. S. 18.

2) Archiv f. Ophthalm. Bd. XXXIV. (3). S. 1. 1888.

3) Die Anomalien der Refraction und Accommodation des Auges; deutsche Ausgabe von Becker, 2. Abdruck. Wien 1880. S. 20.

4) Compt. rend. de la société de biologie. Sér. IX. Tom. V. Nr. 15. 1888. 
hagen seit 1863 vertritt, hat demnach in der letzten Zeit an Boden gewonnen.

\section{Anatomisches über die Irisgefässe.}

$\mathrm{Da}$ ein Theil der Irisbewegungen auf Veränderungen der Irisgefässe zurïckgeführt wird, so ist noch zu erwähnen, inwiefern sich hierfür ein Anhalt in dem Bau der Gefässe vorfindet.

Die Gefässe der Regenbogenhaut sind dureh radiären Verlauf ausgezeichnet. An ibrer Wandung fällt vor Allem die anssergewöhnliche Stärke der Adventitia auf. Auch die Muskelschicht ist nach Arnold ${ }^{1}$ ) besonders ausgebildet. v. Hüttenbrenner beschreibt eine Scheide glatter Muskelfasern um die Gefässe in der Richtung der Längsaxe. Nach Ulrich ${ }^{2}$ ) ist die Längsmusculatur in der Media der kleinen Arterien besonders stark entwickelt und übertrifft die Quermusculatur. Auch Koganeï sah in der Muscularis mehr Iongitudinale, als circuläre Fasern. Auf erstere überträgt $F 1 \mathrm{esch}^{3}$ ) geradezu die Function des Dilatators.

\section{Allgemeines über die Innervation der Iris und über die Pupillenerweiterung und -verengerung.}

Untersuchen wir, ob die Ergebnisse der physiologisehen Forschung ${ }^{4}$ ) mit denen der anatomischen übereinstimmen.

Sämmtliche Irisbewegungen lassen sich erklären, wenn man die Weite der Pupille zuriekfuhrt ${ }^{5}$ ):

1. auf die Stärke der Innervation des M. sphincter pup.;

2. auf den Contractionsustand und die Füllung der Gefässe;

3. auf die Elasticität der Regenbogenhaut.

Gruenhagen legte auf letztere besonderes Gewicht. Unter normalen Verhältnissen darf sie als unveränderliche Grösse angesehen werden. Sie beruht hauptsächlich auf den Scheiden der Nerven und Gefässe und auf der hinteren Grenzlamelle. Diese wird ron $\mathrm{Fuch} \mathrm{s}^{6}$ ) als der lange gesuchte Dilatator betrachtet, indem die durch die

1) Virchow's Archiv. Bd. XXVII. S. 365. 1863.

2) Bericht über die 56. Versamml. deutscher Naturforscher und Aerzte zu Freiburg 1883. S. 255.

3) Vgl. Jahresber. d. Ophthalmol. von Nagel-Michel 1884. S. 8.

4) Ich verweise auf die Zusammenstellung der Literatur bei Budge, Rembold und Leeser.

5) Von Momenten, die nicht in der Iris gelegen sind, lassen sich anführen der intraoculäre Druck, ferner der Contractionszustand des Ciliarkörpers.

6) Lehrbuch der Augenheilkunde. 2. Auf. 1891. 
Kritische u. experiment. Untersuchungen über die Irisbewegungen u. s. w. 97

Contraction des Sphineters gesetzte Spannung des Irisgewebes mit der Ersehlaffung des Muskels zurückgeht.

Die Innervation der Gefässe oder des Sphincters sind dagegen veränderliche Grössen. Es ist daher möglich, dass beide Kräfte sich unterstïtzen oder entgegenarbeiten. Ihre Gleichgewichtsstellung ist so häufigem Wechsel unterworfen.

\section{Pupillenveränderung durch den Lichireiz, durch Atropin und Physostigmin.}

Bei der Netzhautreizung durch Licht verläuft bei Warmblütern die reflectorische Erregung der Iris von den Centralorganen aus nur auf der Bahn des Oculomotorius, denn nach dessen Ausschaltang bleibt bei einem Weehsel der Helligkeit die Pupillengrösse unverändert. Nach Durchschneidung des Trigeminus oder Sympathicus sind die Irisbewegungen nicht anfgeboben. Im Dunkeln fällt natürlich der von der Retina ausgehende Reflex fort. Die Pupillengrösse unter diesen Verhältnissen wurde von Cl. du Bois-Reymon ${ }^{1}$ ) und H. Cohn ${ }^{2}$ auf Magnesiumblitzphotographien von Menschen untersucht. Der Sphincter hat aber auch jetzt noch eine gewisse Bedeutung für die Weite der Pupille, indem Donders ${ }^{3}$ ) es wahrscheinlich macht, dass ein gewisser localer Tonus vom Ganglion ciliare aus selbst nach intracranieller Oculomotoriusdurchschneidung bestehen bleibt. Es kann daher fraglich erscheinen, ob man die Pupillenweite im Dunkeln zweckmässig als Ruhezustand der Iris ${ }^{4}$ ) auffasst.

Die Differenz zwischen der Pupillenweite im Dunkeln und bei einer bestimmten Beleuchtungsintensität stellt eine gewisse Arbeit dar. Letztere behält dieselbe Grösse, mag die Pupille nach Entfernung der Lichtquelle sich dilatiren oder umgekehrt nach einem Aufenthalt im Dunkeln in derselben Intensität beleuchtet werden und sich contrahiren, obschon eigentlich nur letzterer Vorgang als ein activer zu bezeichnen ist. Da ein den Oculomotorius lähmendes Gift bewirkt, dass die Iris das Bestreben hat, eine der vollständigen Ausschaltung jenes Nerven entsprechende Lage einzunehmen, so erfolgt die Pupillenerweiterung mit einem Aufwand an Arbeit, welchen die bei der vorhergebenden Oculomotoriusreizung geleistete sogar noch

1) Centralbl. f. prakt. Augenhl. Bd. XII. S. 68. 1888.

2) Bericht des 7. internationalen Ophthalmologen-Congresses zu Heidelberg. S. 209.1888.

3) a. a. O. S. 498 .

4) Debouzy, A., Considérations sur les mouvements de l'iris. Thèse de Paris 1875 .

A r chiv f. experiment. Pathol, u. Pharmakol. XXX. Bd. 
etwas übertrifft. Infolge dieser Verbältnisse bedarf es sowohl für die durch Atropin, als auch für die durch Oculomotoriusläbmung dilatirte Pupille einer erheblich grösseren Physostigminmenge, um eine starke Myose zu erzielen, als am normalen Auge, da hier die reflectorische Sphinctererregung mitwirkt. Die Physostigminwirkung ist aber auch weniger dauerhaft. Dass hintere Synechien durch Atropin gedehnt und zerrissen werden, lässt sich aus der Oculomotoriuslähmung allein verstehen.') Wenn zwischen zwei Synechien die Iris bisweilen arkadenartig contrahirt erscheint, so ist das noch kein Beweis dafür, dass dieser $\mathrm{Zug}$ in radiärer Richtung auf einer Reizung des Sympathicus durch Atropin beruht. Da die Pupillenweite im Dunkeln und nach Atropininstillation annähernd gleich gross ist, so wird die Wirkung des Atropins durch Aufenthalt im Dunkeln unterstiitzt.

Da beim Menschen auf Netzhautreizung der Sphincter beider Augen sich contrahirt, so sind beide Pupillen gewöhnlich gleich weit. Der gleichmässige Contractionszustand ist zunächst auf diese Ursache zurïckzuführen, wie daraus hervorgeht, dass eine geringe Pupillendifferenz, welche natitrlich nicht auf einer Oculomotoriusparese beruhen darf, durch eine Verstärkung der Beleuchtung ausgeglichen werden kann, wie ich oft beobachtet habe. Zum Verständniss dieser Thatsache ist aber ausserdem nothwendig, sich zu erinnern, dass die Innervation des Sphincter pupillae der des Apparates activer Dilatation in der Iris überlegen ist, denn wenn bei Thieren der N. oculomotorius zugleich mit dem Halstheil des N. sympathicus elektrisch gereizt wird, so verengt sich die Pupille. ${ }^{2}$ )

Thiere mit mangelhaft entwickeltem binocularen Sehen lassen eine grössere Unabhängigkeit der Bewegungen jeder der beiden Regenbogenhäute erkennen. Von Kaninchen ist bekannt, dass auf Lichteinfall nur auf der betreffenden Seite die reflectorische Myose sich einstellt. ${ }^{3}$ )

\section{Besondere Arten der reflectorischen Pupillenerweiterung.}

Für die Beurtheilung der Irisbewegungen ist noch ein anderer Reflexvorgang zu berücksichtigen, welcher in einer Beziehung zam Oculomotorius steht. Sehr complicirter Natur sind gewisse reflectorische Dilatationen der Pupillen, welche in der verschiedensten Weise entstehen können.

1) Vgl. Eversbusch, Zeitschr. f. vergl Augenheilk. Bd. III. S. 40 u. 43.1875.

2) Vgl. Landois, Lehrb. d. Physiologie des Menschen. 5. Aufl. 1877. S. 867.

3) Vgl. Luchsinger, Archiv f. d. ges. Physiol. Bd. XXXIY. S. 294. 1884. 
a) Pupillenerweiterung durch psychische Einflüsse.

Fontana ${ }^{1}$ ) war vielleicht der Erste, welcher eine derartige Beobachtung anstellte. Er fand, dass die Augen einer in Schrecken versetzten Katze weite Pupillen bewahrten, selbst wenn intensives Licht einfiel. Bei Kussmaul ${ }^{2}$ ) finde ich die Angabe, dass Alles, was die Thiere erschreckt, Geräusche, Erschütterungen, Mydriasis macht. Anch Gratiolet ${ }^{3}$ ) erwähnt diese Pupillenerweiterung nach psychischen Eindrücken infolge heftiger, unerwarteter Gehörseindrücke.

Auf den Wechsel der sensiblen und psychischen Eindrücke bezogen Schadow ${ }^{4}$ ), Rieger und v. Forster ${ }^{5}$ ) und Laquea ${ }^{6}$ ) von Letzterem als Pupillenunruhe bezeichnete Schwankungen der Pupille. ${ }^{7}$ )

b) Pupillenweite bei Reizung sensibler Nerven, besonders des Trigeminus und der Grosshirnrinde.

Cl. Bernard ${ }^{8}$ ) giebt eine genaue Schilderung von der reflectorischen Dilatation beider Pupillen nach Reizung der verschiedensten Nerven. Weiter beschäftigten sich mit dem Gegenstande Westphal ${ }^{9}$ ), Wegener ${ }^{10}$, Hecker ${ }^{11}$ ), Vulpian ${ }^{12}$ ), Schiff and Foa ${ }^{13}$ ), Debouzy, Holmgren ${ }^{14}$ ), Hurwitz ${ }^{15}$ ), Bessau ${ }^{16}$ ), Rembold ${ }^{17}$ ) u. A.

1) Citirt nach Budge a. a. O. S. 165.

2) Untersuchungen über den Einfluss, welchen die Blutströmung auf die Bewegungen der Iris und anderer Theile des Kopfes ausübt. Inaug.-Diss. Würzburg 1855.

3) De la Physionomie et des mourements d'expression 1865. (Biblothèque d'éducation et de récréation. p. 256.)

4) Archiv f. Ophthalm. B. XXVIII (3). S. 188. 1882.

5) Ebenda. Bd. XXVII (3). S. 182. 1881.

6) Klin. Monatsblatt f. Augenheilk. Bd. XXV. S. 463.1887.

7) Vgl. S. 102.

8) Journal de la physiol. V. 1862.

9) Virchow's Archiv. Bd. XXVII. S. 409. 1863.

10) Archiv f. Ophtbalm. Bd. XII (2). 1866.

11) Tagebl. d. 45. Persamml. d. Naturf. u. Aerzte in Leipzig. 1872. S. 152.

12) Archives de physiol. norm. et path. 1874. - Leçons sur l'appareil vasomoteur. Paris 1875. T. I. p. 293. - Gaz. méd. 1878. No. 27. p. 328.

13) La pupille considérée comme esthésiomètre. Traduction de l'italien par R. Guichard de Choisity. Paris 1875.

14) Citirt nach Jahresber. d. Ophthalm. von Nagel-Michel 1876. S. 158.

15) Ueber die Reflexdilatation der Pupille. Inaug.-Diss. Erlangen 1878.

16) Die Pupillenenge im Schlafe und deren Bedeutung bei den Krankheiten des Centralnervensystems. Inaug.-Diss. Königsberg 1879.

17) Ueber Pupillarbewegung und deren Bedeutung bei den Krankheiten des Centralnervensystems. Mittheilungen aus der ophth. Klinik in Tübingen von A. Nagel. Bd. I (2). 1880. 
Der Erfolg bei Reizung von Trigeminusästen wird verschieden angegeben. Cl. Bernard, sowie Leeser ${ }^{1}$ ) und Hecker saben dabei ebenfalls Dilatation. Debouzy und Rembold beschrieben bei directer Hornhautreizung erst kurzdauernde Erweiterung und hierauf Verengerung. Ich habe mich davon überzeugt, dass die Pupille sich hierbei vorübergehend erweitern kann. Von der Nasensehleimhaut aus erhielt J. Müller ${ }^{2}$ ) nur Myose, bei directer Hornhautreizung auch Hurwitz. Die Pupillenerweiterung, welche $\mathrm{Chauveau}^{3}$ ) bei der Reizung der Wurzeln der Rückenmarksnerven in der Gegend des Centrum cilio-spinale von Budge erbielt, ist ebenfalls anzuführen.

Auch von der Grosshirnrinde aus hat man ähnliche Phänomene an den Augen hervorgerufen. Schiff und Foa, Bochefontaine ${ }^{4}$ ), Luciani und Tamburini ${ }^{5}$ ), Gruen hagen ${ }^{6}$ ), Bessau, Francois-Franck fanden als Resultat eine Erweiterung der Pupillen. Diese lässt sich bei Kaninchen nach Bessau am leichtesten vom Occipitallappen auslösen. Wurde zuerst der Frontallappen und unmittelbar darauf der hintere Abschnitt der Gehirnoberfläche gereizt, so blieb der Erfolg aus. Brown-Séquard ${ }^{8}$ ) erhielt nach Cauterisation der Gehirnoberfläche mit dem Glüheisen Myosis, welche er auf eine Lähmung des Halssympathicus bezieht. Die Papille verengerte sich bisweilen erst etwas nach der Zerstörung des Gehirns, regelmässig, wenn das Glüheisen in hinreichender Ausdehnung angewandt wurde. Die Myose nahm während der folgenden Stunden noch zu. In einem Falle war die Pupille des Kaninchens unmittelbar nach einer geringen Verbrennung des Grosshirns weit. $\mathrm{Ob}$ auch bei den anderen Thieren eine Dilatation vorausging, ist nicht ersichtlich. Jedenfalls dürfte den von Brown-Séquard nach diesen meist ausgedehnten Zerstörungen des Grosshirns beobachteten Erscheinungen eine andere Bedeutung zukommen. ${ }^{9}$ )

1) Die Pupillarbewegung in physiologischer und pathologischer Beziehung. Wiesbaden 1881. - Beiträge zur Physiologie der Pupillarbewegung. Inaug.-Diss. Halle 1881.

2) Handb. d. Physiologie des Menschen. 4. Aufl. 1844. Bd. I. S. 613 u. 614.

3) Comptes rendus 1861. II. p. 581. - Journ. de la physiol. IV. p. 360. 1861.

4) Comptes rendus. T. LXXXIII. p. 233. 1876. - Archives de physiol. norm. et path. T. VI. p. 15. 1879.

5) Citirt nach Jahresbericht d. Ophthalm. von Nagel u. Michel. 1879.

6) Berl. klin. Wochenschr. 1879. S. 407.

7) Gaz. hebdom. No, 32 u. 33. 1888.

8) Archives de physiol, norm. et path. T. II. p. 854. 1875.

9) Ha a b (Der Hirnrindenreflex der Pupille. Zürich 1891) hat kürzlich eine eigenthümliche reflectorische Pupillenverengerung beschrieben, bei welcher die Aufmerksamkeit eine Rolle spielt. 
Kritische u. experiment. Untersuchungen über die Irisbewegungen u. s. w. 101

c) Innervationsverhältnisse bei den reflectorischen Irisbewegungen.

Die reflectorische Mydriasis bezog man zunächst auf einen die Pupille dilatirenden Muskel. Cl. Bernard sah sie nicht mehr, wenn die oculopupillären Nerven infolge Durchschneidung der betreffenden Rückenmarkswurzeln ausgeschaltet waren. Auch nach Schiff hebt Durchschneidung des Halssympathicus die Reflexdilatation bei Reizung sensibler Nerven und des Grosshirns auf. Die Wichtigkeit der letzteren geht aber doch schon daraus hervor, dass nach Exstirpation beider Hemisphären sensible Reize bei Katzen und Hunden keine Erweiterung der Pupille mehr bewirkten, während die Irisbewegungen auf Einfluss des Lichtes und der Augenbewegungen erhalten waren.

Andere Beobachter fanden, dass der Halssympathicus keinen wesentlichen Einfluss auf das Zustandekommen dieser Mydriasis ausuibt. Nach Durchschneidung des Halsmarkes, wie Salkowski ${ }^{1}$ ) angiebt, oder nach Exstirpation des obersten Halsganglions, wie Vulpian, Hurwitz, Bessau fanden, ist die Pupillenerweiternng anf Reizung peripherer Nerven oder der Grosshirnoberfläche (Bochefon taine, Bessau) nicht aufgehoben. Bei Katzen ist nach einigen Tagen die Reflexdilatation auf der operirten Seite sogar stärker als auf der normalen. Die Pupille gewinne an Dilatationsvermögen, da die verengernde Kraft des Sphincters eine Einbusse erleide ( $\mathrm{Hu} r \mathrm{r}$ itz). Das Letztere wird man wohl als eine secundäre Erscheinung auffassen. Vulpian, Bochefontaine, Bessau, Leeser, Rembold glauben zu der Annahme berechtigt zu sein, dass nicht alle pupillenerweiternden Nerven des Halsstranges den Sympathicus oder das oberste Halsganglion durchziehen. Der anatomische Nachweis dieser Fasern ist nicht erbracht. Physiologische Thatsachen lassen sich für ihre Existenz nicht weiter anführen. Ein solcher Schluss ist aber auch deshalb anfechtbar, weil die Möglichkeit vorliegt, dass eine Hemmung jenes Reflexes durch den psychischen Reiz im Spiele ist, welcher von der durch die Lichtstrahlen gereizten Netzhaut zum Oculomotorius gelangt ( $\mathrm{Hurwitz}, \mathrm{Plotke}{ }^{2}$ ). Das angeführte Experiment von Fontana am Katzenauge ist auf diese Weise leicht zu verstehen, da die Innervation des Sphincters der des "Dilatators" überlegen ist. Nach Bechterew ${ }^{3}$ ) ist die Pupillenerweiterung infolge von Schmerz bei heller Beleuchtung deutlicher als bei herab-

1) Zeitschr. f. rat. Med. Bd. XXIX. S. 167, 1867.

2) Archiv f. Psychiatrie. Bd. X. S. 205. 1880.

3) Archiv f. die ges. Physiol. Bd. XXXI. S. 60. 1883. 
gesetzter. $\mathrm{Ob}$ in den Versuchen von $\mathrm{Ch}$ auvean der Sympathicus die Bahn darstellte, steht meiner Ansicht nach nicht ohne Weiteres fest. Wie weit die besprochene Pupillenerweiterung durch psychische Einfliusse ausschliesslich auf einer Hemmung der Sphinctercontraction beruht, ist noch nicht erwiesen, denn es muss als wahrscheinlich gelten, dass für einige dieser Erscheinungen ausserdem wenigstens theilweise der "Dilatator iridis" die Ursache bildet. Hierfür spricht, dass gleichzeitig Blutdruckschwankungen (Schiff, Hurwitz) bestehen.

Eine Bedeutung der Exstirpation des Ganglion cervicale wurde nicht nur von den Untersuchern beobachtet, welche die Erscheinungen durch den Sympathicus vermitteln wollen. So giebt Bessau an, dass bei einer Katze, welcher der Sympathicus einseitig am Halse zerstört worden war, auf Reizung der Grosshirnhemisphären nur die Pupille des normalen Auges sich erweiterte, wenn der Inductionsstrom nicht verstärkt wurde.

\section{Einfluss der Gefässfüllung auf die Pupillenweite.}

Einen Einfluss der Blutgefässe auf die Irisbewegungen nahm schon Haller an. Die Myose nach Abfluss des Kammerwassers wird auf eine Steigerung des Blutgehaltes der Regenbogenhaut bezogen. Mittelst Injection der Blutgefässe gelang es Caddi ${ }^{1}$ ), Rou $\mathrm{get}^{2}$ ), Mosso ${ }^{3}$, an todten Thieren Pupillenverengerung hervorzurufen. Brown-Séquard ${ }^{4}$ ) und $W$ eischer konnten allerdings auf diesem Wege eine nennenswerthe Veränderung der Iris nicht nachweisen, wohl weil es schwierig ist, wie Leber ${ }^{5}$ ) betont, die Gefässe der Iris gleichmässig mit Injectionsmasse anzufüllen.

Hensen und Völckers ${ }^{6}$ ) beobachteten unbedeutende, mit Puls und Respiration synchron verlaufende Schwankungen der Pupillenweite, welche von ihnen auf die Blutströmung zurückgeführt werden. Diese Bewegungen des Pupillarrandes lassen sich besonders gut mit der Binocularloupe wahrnehmen. Wenn Brown-Séquard Thiere

1) Gaz. médic. 1846.

2) Compt. rend. et mém. de la société de biologie. 1856. p. 130.

3) Citirt nach Jahresber. d. Ophthalm. von Nagel-Michel. 1875.

4) Gaz. méd. de Paris 1849. p. 744 u. Ann. d'oculistique. T. XXII. 1849. p. 165.

5) Vgl. Handbuch d. ges. Augenhk. von Gräfe und Sämisch. Bd. II. 1876. S. 320 u. 362 .

6) Experimentaluntersuchung über den Mechanismus der Accommodation. Kiel 1868. - Ferner Coccius, Ophthalmometrie und Spannungsmessung am kranken Ange. Leipzig 1872, citirt nach $\mathrm{Nagel}$, Jahresber. d. Ophth. von NagelMichel. Bd. III. 1872. S. 139. 
Kritische u. experiment. Untersuchungen über die Irisbewegungen u. s. w. 103

an den Hinterbeinen aufhing, so verengerten sich die Pupillen. De bouzy bestätigte dasselbe für den Menschen. Es ist möglich, dass diese Myose auf einer vermehrten Gefässfüllung beruht. Während die von Kussmaul an der Pupille beobachteten Phänomene im Allgemeinen auf eine durch die gewaltigen Eingriffe herbeigeführte Reizung der Centralorgane bezogen werden können, so ist die Pupillenerweiterung, welche in jenen Versuchen nach Blutentziehungen aus den Halsvenen eintrat, vielleicht durch eine Verminderung des Blutgehaltes zu erklären. Wernicke ${ }^{1}$ ) stellte ähnliche Experimente an, welche ebenfalls nicht eindeutig sind.

Neben der Blutmenge ist auch der Contractionszustand der Gefässwandung zu berücksichtigen. Es geht dies daraus hervor, dass die von Biffi gefundene Pupillenerweiterung nach Reizung des Halssympathicus auch an todten Thieren und am isolirten Kopfe erreicht wird.

Wenn ein besonderer M. dilatator pupillae nicht existirt, so ist die strenge Unterscheidung von oculopupillären und vasomotorischen Sympathicusfasern durch Cl. Bernard ${ }^{2}$ ) unwesentlich. Die topographische Sonderung gelang ihm nur beim Hunde, für das Kaninchen wird sie wenigstens von Salkowski ${ }^{3}$ ) bestritten. Indem die Bewegungsveränderungen der Papille gleichzeitig mit Schwankungen im Lumen der Gefässe der Iris und des Augenhintergrundes auftreten, wie ich bestätigen kann, so liegt es nahe, beide Erscheinungen im Zusammenhang zu besprechen. In letzter Zeit machten hauptsächlich Gruenhagen und Eversbusch den Versuch, die oculopupillären Symptome in grössere Abhängigkeit von den Gefässveränderungen zu bringen.

\section{Verhalten der Pupille im Schlaf.}

Die Beschaffenheit der Iris im Schlafe wird in hohem Maasse durch das Verhalten der Gefässe bestimmt. Fontana machte nach Ruete ${ }^{4}$ ) zuerst darauf aufmerksam, dass die Pupille im Schlafe verengt ist. Derselbe stellte auch bereits Versuche an bei Katzen und Menschen. Aus neuerer Zeit stammen Arbeiten von Rählmann u. Witkowski ${ }^{5}$ ),

1) Virchow's Archiv. Bd. LVI. S. 397. 1872.

2) Compt. rend. T. XXXIV. p. 472. T. XXXVI. p. 375. - Journ. de la physiol. T. V. p. 383. 1862.

3) Zeitschr. f. rat. Med. Bd. XXIX. S. 167.

4) Lehrbuch der Ophthalmologie. 2. Aufl. Bd. I. 1853. S. 97.

5) Archiv f. Anat. u. Physiol. 1877 und 1878; ferner Archiv für Psychiatrie. Bd. IX. S. 443, 1879. 
Siemens ${ }^{1}$, Sander ${ }^{2}$, Rembold, Plotke u. A. Dieselben ergaben, dass die Myose mit der Tiefe des Schlafes zunimmt und selbst maximal wird. Wie im wachen Zustande reagirt die Iris auf Lichteinfall; bei maximaler Verengerung vermisste Plotke diese Bewegung. Die Pupille erweitert sich ferner auf sensible und akustische Reize und zwar um so ausgiebiger, je weniger tief der Schlaf ist (Westphal). Auf dieselbe Ursache ist die starke Erweiterung beim Erwachen zurïckzuführen, welche schon $J$. Mỉller ${ }^{3}$ ) bekannt war. Wenn man die Lider eines Schlafenden öffuet, so hat man öfters die Pupillen verhältnissmässig weit gefunden. Dies ist vielleicht ebenso zu erklären. Die Pupillen können hierauf wieder enger werden, sei es durch das einfallende Licht, sei es durch die mit dem Schlafe selbst verbundenen Erscheinungen.

Zur Erklärung dieser Thatsachen hat man verschiedene Ansichten aufgestellt. J. Muller fasste die Myose als Mitbewegung auf, indem die Augenaxen nach innen und oben gerichtet seien. Gegen diese Anschaung wandte sich $\mathrm{Ruete}$, da er fand, dass die Augenaxen auch andere Stellungen einnehmen können und die Pupillen auch bei Lähmung oder Durchschneidung des Rectus internus contrahirt sind. Rählmann und Witkowski u. A. zeigten, dass bei Schlafenden mannigfaltige Bewegungen vorkommen. Diese gehen vollständig unregelmässig und selbst monocular vor sich. Die Pupillen bleiben hierbei unverändert. Von einer Mitbewegung im Sinne von J. Müller kann also nicht die Rede sein. Sander glaubt, dass die Augen im Schlafe eine mittlere Stellung mit parallelen Augenaxen einnehmen. Die Muskeln befänden sich demnach in der Ruhelage, und die Augen nehmen eine Stellung ein, wie es den einzelnen Kräften entspricht. Ist der Schlaf nicht ganz fest, und man hebt die oberen Augenlider in die Höhe, so wenden sich die Bulbi nach oben und innen, um dem einfallenden Lichte zu entfliehen. Es scheint mir, dass Bewegungen der Augen im Schlafe nur insofern möglich sind, als Contractionen willkürlicher Muskeln während desselben iiberbaupt zugelassen werden. Im festen Schlafe dürften sie fehlen.

Si em en s und Sander vermuthen, dass die Pupillenverengerung: im Schlafe auf eine directe centrale Innervation zurückzufibren sei.

1) Archiv f. Psychiatrie. Bd. IX. S. 72. 1879.

2) Ebenda. Bd. IX. S. 129. 1879. - Berliner klin. Wochenschrift. Nr. 46. S. 652. 1876 .

3) A. a. O. Bd. II. S. 583. 
Kritische u. experiment. Untersuchungen über die Irisbewegungen u. s. w. 105

Verschiedene Theorien(E.H. Weber ${ }^{1}$ ), Ritterich ${ }^{2}$ ), Gruenhagen, Bessau, Plotke) nehmen einen selbständigen Reizzustand des M. spincter pup. an. Weber zieht den Vergleich mit anderen Organen, welche einen Schliessmuskel besitzen. Da aber auch bei Amaurotischen die Pupille sich contrahirt ( $\mathrm{Ru}$ e te), so kann die Iris die Function nicht besitzen, die Lichtstrahlen abzuhalten. Da nach Gruenhagen, Bessau, Plotke auch das atropinisirte Ange im Schlafe eine weite Pupille besitzt, so geht hieraus jedenfalls hervor, dass ein Theil der beobachteten Myose auf eine Sphincterreizung zu schieben ist. Eine andere Frage ist es natürlich, ob nicht hierfür die Umstände, unter denen untersucht worden ist, verantwortlich zu machen sind. Um die Iris Schlafender zu besichtigen, ist eine ziemlich intensive Beleuchtung erforderlich. Diese bildet nothwendig: einen starken Reiz für das in der Dunkelheit ausgeruhte Auge. Auf eine solche Netzhatreizung muss meiner Ansicht nach ein Theil der Pupillenenge bezogen werden. Sicheren Aufschluss könnte vielleicht eine photographische Aufnahme bringen. Nach Ruete wird die Ursache der stärkeren "Contraction des Sphincters" im Schlafe durch die gesunkene Empfindlichkeit der sensiblen Nerven gebildet, indem nach Durchschneidung des Trigeminus die Pupille eng wird.

Rählmann und Witkowski führen den Zustand der Iris im Schlafe darauf zurück, dass der Reiz zur Erweiterung feble. Die zahlreichen sensiblen, sensoriellen und psychichen Erregungen, welche auf den Wachen einstürmen, fehlen im Schlafe fast vollständig. Man könne hiergegen einwenden, dass ein Reiz nur dann, wenn er erscheint oder aufhört oder eine Steigerung erfährt, eine solche Reflexdilatation hervorbringt. Auch liesse sich die Weite der Pupille im Dunkeln anführen. Die Bedeutung der gesunkenen Reflexempfindlichkeit ist in ihrem Einfluss auf den Tonus der Gefässe zu suchen. Nach Rembold ist durch die Annahme eines Musculus dilatator eine Erklärung nicht möglich, denn bei einer Lähmung zweier entgegengesetzt wirkender Muskelapparate muisse eine mittlere Pupillenweite resultiren. Da ein Reizzustand des Oculomotorius nicht anzunehmen ist, so muss die Pupillenverengerung auf eine Erschlaffung der Gefässwand zurückgeführt werden, welche eine stärkere Füllung mit Blut bedingt. Eine Aenderung in dem Blutgehalt peripherer Gefässgebiete während des Schlafes ist zu beobachten und besonders von

1) De moto iridis. 1821.

2) Weitere Beiträge zur Vervollkommnung der Augenheilkunst. Leipzig 1861. Citirt nach Rembold. 
der Conjunctiva bekannt (Plotke, Sander). Ich kann aber nicht darin mit Rembold iibereinstimmen, dass diese Pupille als Rubelage zu betrachten sei. Der Tonus der Gefässe hat im Schlafe zwar nachgelassen, ist aber nicht vollständig aufgehoben.

\section{Pupillenweite in der Narkose.}

Der künstlich herbeigefübrte Schlaf, die Nark ose, hat mit dem natürlichen eine gewisse Aehnlichkeit hinsichtlich der an der Pupille sichtbaren Phänomene. Beim Menschen gestattet die Pupillengrösse directe Schlüsse auf die Tiefe der Narkose. Simpson ${ }^{1}$ ) giebt bereits an, dass die Pupillen wäbrend der Chloroformirung bald weit, bald verengt oder unverändert sind. $\mathrm{Ruete}{ }^{2}$ ) unterscheidet bereits die drei Stadien. Im ersten, gleichzeitig mit der Aufregung, sind die Pupillen weit. Sie contrahiren sich in tiefer Narkose, in welcher die willkürlichen Bewegungen aufhören und die Sensibilität vollständig erloschen ist. Ein Nachlass der Myose bedeutet entweder die Wiederkebr der Empfindung und des Bewusstseins, oder aber bei noch bestehender Insensibilität ist sie das Zeichen der Asphyxie. Der eben beschriebene Verlauf der Irisbewegungen trifft mit grösster Regelmässigkeit für die Chloroform- und Aethernarkose beim Menschen zu. Für andere Glieder der Chloral- und Alkoholgruppe lauten die Angaben nicht gleichmässig. ${ }^{3}$ )

Die Thierversuche widersprechen sich noch mehr. Einen ähnlichen Verlauf der Chloroformnarkose wie beim Menschen sahen Gruenhagen, Budin und Coyne ${ }^{4}$, Fischer ${ }^{5}$ n. A. Rembold ${ }^{6}$ ) beobachtete bei Kaninchen eine mittlere Weite der Pupillen. Diese werden unmittelbar vor dem Tode enger und dilatiren sich hierauf stark. Dass während der Narkose die Pupillen erst verengt, dann weit seien, behaupten Dogie ${ }^{7}$ ) für Chloroform und Panb off ${ }^{8}$ ) für Methylenchlorid. Besonders abweichende Angaben machen Schiff und Foa, nach denen die Pupillen chloroformirter Hunde fast ausschliesslich erweitert sind, während Chloral Myose erzeugt. Gegen

1) The monthly journal of medical science. Vol. VIII. 1847. Dec. S. 415.

2) a. a. O. S. 99.

3) Harnack, E., Lehrb. der Arzneimittellehre. 1883. S. 563, 575, 582, 592.

4) Le progrès médicale 1874. No. 36. p. 525. - Gaz. méd. de Paris 1875. p. 67 et 90. - Archives de physiol. norm. et path. 2. s. II. p. 61.1875.

5) Archiv f. klin. Med. Bd. XVII. S. 1. 1875.

6) Mittheilungen aus d. ophth. Klinik in Tübingen von A. Nagel. Bd. I (1). 1880. S. 221 .

7) Arch. f. Anat. u. Physiol. 1866. S. 231.

8) Ueber die physiol. Wirkung des Methylenchlorid. Inaug.-Diss. Erlangen 1882. 
Kritische u. experiment. Untersuchungen über die Irisbewegungen u. s. w. 107

diese Autoren wandten sich Budin und Coyne, indem sie dem Chloral sowohl, als auch dem Chloroform eine pupillenverengernde Wirkung zuschreiben und die von Schiff und Foa beschriebenen Erscheinungen für ein Gemenge von Anästhesie und Asphyxie halten.

Wie im Schlafe reagirt die Pupille im Beginn der Narkose auf Belichtung (Hirschberg ${ }^{\dagger}$ ) und auf Reizung peripherer Nerven und auf starke Gehörseindrücke (Westphal, Holmgren, Budin und Coyne, Sehiff und Foa, Rembold u. A.). Nach Schiff zeigt sich Pupillenerweiterung nach Reizen, die noch kein Anwachsen des Blutdruckes hervorbringen. Vom Rückenmark aus soll sogar eine Steigerung des Blutdruckes sich erreichen lassen, ohne dass die Pupille sich dilatirt. Diese Angaben Schiff's werden von Hurwitz nicht bestätigt. In vollständiger Narkose fehlen dagegen die reflectorischen Bewegungen der Pupillen, selbst elektrische Reizung des $\mathrm{N}$. opticus ist erfolglos (Dogiel).

Hinsichtlich der Erklärung der Reflexdilatation kann ich wohl auf die früheren Auseinandersetzungen verweisen.

Die Myose, welche in tiefer Narkose sich einstellt, wurde auf versiedene Ursachen zurtickgeführt. Pannh off glaubt, dass bei der Methylenchloridnarkose eine Reizung des Oculomotorius stattfinde, dessen Tonus im weiteren Verlaufe nachlasse. Es muss darauf hingewiesen werden, das Chloroform, Aether, Chloralhydrat u. s. w., wenn man von einer localen Reizung absieht, rein lähmend wirken. ${ }^{2}$ ) Eine directe centrale Reizung durch irgend ein Glied der Alkoholgruppe ist nicht erwiesen. Eine solche Einwirkung auf den Oculomotorius oder Sphincter pup. darf auch deshalb ausgeschlossen werden, weil Reizung des Oculomotorius während der Narkose nach Dogiel noch wirksam ist, und weil die atropinisirte Pupille in der Chloroform- (Gruenhagen) und Chloralnarkose (Ulrich ${ }^{3}$ ) sich verengt. Periphere in der Iris gelegene Apparate scheinen nicht wesentlich betheiligt zu sein. Reizung des Sympathicus giebt das gewöhnliche Resultat (Dogiel). Trigeminusdurchschneidung verhindert die Myose nicht (Gruenhagen). Bei der directen elektrischen Hornbautreizung erhielt $D_{0}$ giel keine vom Normalen abweichenden Resultate, mochten die Elektroden dem äusseren oder inneren Irisrand entsprechend aufgesetzt werden. Da die Iris selbst nicht beeinträchtigt erschien, so dachte Dogiel an eine Störung der cen-

1) Berliner klin. Wochenschr. 1876. Nr. 45. S. 652.

2) Vgl. v. d. Mü hIl und Jaqu et, Correspondenzblatt für die Schweizer Aerzte. 1891.

3) Archiv f. Ophthalm. Bd. XXVI (3). S. 35. 1880. 
tralen Functionen in der Weise, dass entsprechend den drei Stadien der Chloroformvergiftung erst eine Erregung, dann eine Parese und hierauf eine Lähmung von Centren eintreten soll. Eine primäre Erregung besteht nicht. Ferner trifft der von Dogiel beschriebene Symptomencomplex für den Menschen nicht zu und wird auch bei Thieren nicht regelmässig beobachtet.

Eine befriedigende Erklärung muss in Gefässveränderungen gesucht werden.1) Auf die Blutgefässe wirkt die Chloralgruppe in der Weise, dass neben einer Abschwächung der Herzthätigkeit eine Lähmung der Gefässnervencentren sich einstellt. Sehr früh werden besonders die Gefässe des Gesichts and Gehirns betroffen. ${ }^{2}$ ) Die Röthung des Gesichts beim Menschen schwindet mit der Lähmung anderer Gefässbezirke. Diese Verbältnisse geben eine genügende Erklärung für die uns beschäftigende Myose. Der Tonus der Irisgefässe, welcher während des Schlafes wahrseheinlich schon vermindert ist, lässt durch die Narkose noch mehr nach. Die erschlaffte Gefässwandung kann eine grössere Blutmenge aufnehmen; die Pupille wird eng. Indem bei fortschreitender Narkose die Lähmung sich auf weitere Gefässprovinzen ausdehnt, so kann die Anfüllung der Kopfgefässe mit Blut abnehmen. Wenn es richtig ist, dass die Myose auf den vergrösserten Blutgehalt zu beziehen ist, so muss sie mit der Abnahme derselben geringer werden. Da der Mensch und die versehiedenen Thierarten sich nicht gleichmässig verhalten und die einzelnen Substanzen der Chloral- und Alkoholgruppe auch Unterschiede hinsichtlich der Schnelligkeit und vielleicht anch der Reibenfolge der Gefässnervenlähmung erkennen lassen, so ist es verständlich, dass die Thierversuche nicht vollständig übereinstimmen. Diese Erwägungen zeigen ferner, ob man die enge Pupille während der Narkose als Zustand der Ruhe und Passivität (Rembold) ansehen darf. Für die Ziele, welche verfolgt werden, mag eine solche Annahme vielleicht zweckmässig sein, zumal wenn man den Menschen allein im Auge hat. Andererseits ist aber zu bedenken, dass derselbe Zustand der Irisgefässe eine verschiedene Weite der Pupille zur Folge haben kann, indem letztere in einer gewissen Abhängigkeit von dem Tonus der Gefässe des ganzen Körpers stehen. Es dürfte auch nicht leicht sein, den Grad der Lähmung der Irisgefässe zu beurtheilen.

Die weite Pupille des dritten Stadiums scheint wenigstens theilweise auf einer centralen Reizung des Sympathicus zu beruben, denn

1) Vgl. Koch, W., Ueber das Chloroform und seine Anwendung in der Chirurgie. Samml. klin. Vortr. von Volkmann. Nr. 80.

2) Schmiedeberg, 0., Grundriss d. Arzneimittellehre. 2. Aufl. S. 16. 1888. 
Kritische u. experiment. Untersuchungen über die Irisbewegungen u. s. w. 109

nach Exstirpation des Ganglion cervic. supr. fehlte sie (Gruenhagen und $\operatorname{Cohn}^{1}$ ), Schiff ${ }^{2}$ ).

\section{Uebersicht verschiedener Giftwirkungen am Auge.}

In einen gewissen Gegensatz zu den eben besprochenen Erscheinungen können andere gebracht werden, welche dadurch ausgezeichnet sind, dass sie bei localer Application von Giften in den Bindehautsack eintreten.

Die meisten dieser Gifte afficiren ausschliesslich den N. oculomotorius. Eine Gruppe, als deren Hauptrepräsentant das A tro p in ${ }^{3}$ ) betrachtet werden kann, lähmt ohne vorausgehende Steigerung der Erregbarkeit die Endigungen dieses Nerven, die Pupille wird erweitert. Genau die entgegengesetzte pharmakologische Bedeutung: kommt dem Muscarin ${ }^{4}$ ) zu. Wegen der starken Erregung der Endorgane des N. oculomotorius entsteht stärkste Myose. Dem Muscarin ähnlich verhalten sich Pilocarpin und Nicotin ${ }^{5}$ ), doch folgt oft auf die Erregung eine Abnahme der Erregbarkeit der Nerven, welche sich als geringfiigige Dilatation der Pupille kundgiebt. Eine Reizung: des M. sphincter pupillae selbst übt das Physostigmin ${ }^{6}$ )aus. Die Wirkung dieser verschiedenen Substanzen zeigt das bekannte Experiment ${ }^{7}$ ): Die Muscarinmyose wird durch geringe Dosen Atropin aufgehoben und in starke Mydriasis übergefiuhrt; duch Physostigmin lässt sich wieder eine starke Verengerung erzielen. Dieser Versuch lässt sich noch erweitern, indem man vor dem Muscarin Cocain einträufelt. Die hierdurch entstehende Pupillenerweiterung wird durch das Alkaloid des Fliegenpilzes aufgehoben. Die Genese der Mydriasis durch Cocain weicht nämlich wesentlich von der durch Atropin bedingten ab. Da man Ersterem eine directe Reizung des dilatirenden Apparates in der Iris zuschreiben muss, so ist eine Beschäftigung mit dem. selben ganz besonders geeignet, um Aufklärung iuber die Existenz eines selbständigen Dilatators zu verschaffen.

1) Centralbl. f. prakt. Augenheilkunde. 1884. S. 165.

2) Citirt nach Zeitschr. f. rat. Med. Bd. XXXV. S. 477.

3) Siehe Harnack, Arch. f. exp. Path. u. Pharm. Bd. II. S. 307. 1874.

4) Schmiedeberg u. Koppe, Das Muscarin, das giftige Alkaloid des Fliegenpilzes. Leipzig 1869.

5) Siehe Harnack u. Meyer, Archiv für exp. Path. u. Pharm. Bd. XII. S. 366. 1880 .

6) Siehe Harnack u. Witkowski, Ebenda. Bd. V. S. 401.1876 und Har nack, Ebenda. Bd. XII. S. 336. 1880.

7) Siehe Schmiedeberg, 0., Grundriss der Arzneimittellebre. 2. Aufl. S. 81. 1888 . 
10. Ueber die Pupillenerweiterung durch Cocain.

Cocain wurde 1860 von Niemann ${ }^{1}$ ) aus den Blättern der Erythroxylon Coca dargestellt. Schroff ${ }^{2}$ ), welcher bald darauf die physiologischen Eigenschaften des neuenideckten Alkaloides an Warmbliitern untersuchte, fand bereits die dilatatorische Wirkung auf die Pupille. Sie trat sowohl bei Allgemeinvergiftung, als bei localer Anwendung ein. Diese Angabe wurde durch Fronmüller ${ }^{3}$ ) u. A. bestätigt. Bei Kaltblütern beobachtete die Mydriasis zuerst Moreno y Maïz. ${ }^{4}$ Es folgten dann Untersuchungen ron Nikolsky ${ }^{5}$, Danin $0^{5}$ ), v. Anrep ${ }^{6}$ ) a. A. Nach der Empfehlung des Alkaloides durch Koller ${ }^{7}$ ) erschienen zahlreiche Arbeiten, welche besonders uiber die Erscheinungen am Auge handeln.

a) Die Pupillenerweiterung durch Cocain verglichen mit der durch Atropin.

Als besondere Eigenthümlichkeit der Cocainpupille wird angegeben, dass die Dilatation niemals maximal ist (v. Anrep u. A.), indem sie hinsichtlich der W eite hinter der Atropinmydriasis zurücksteht. Unter den gewöhnlichen Versuchsbedingungen ist dies für den Menschen allerdings richtig. Dass Ausnahmen vorkommen, geht schon aus einer Beobachtung von $\mathrm{E}$. $\mathrm{Pfl}$ ï g e ${ }^{8}$ ) hervor, welcher beim Schaf durch Cocain eine stärkere Erweiterung der Pupille erreichte, als durch Atropin. Bei Kaninchen, an denen ich meine Untersuchungen anstellte ${ }^{9}$ ), ist es sogar die Regel, dass Cocain eine bedeutendere Mydriasis hervorbringt, als Atropin. Der Unterschied beträgt mehrere Millimeter. Ich kann daher die entgegengesetzte Behauptung von Berthold ${ }^{10}$ ) nicht bestätigen.

Die Ungleichwerthigkeit der Atropin- und Cocainmydriasis geht ferner daraus hervor, dass die cocainisirte Pupille durch

1) Vierteljahrschrift f. prakt. Hharmacie. Bd. IX. 1860.

2) Wochenbl. d. Zeitschr. d. k. k. Ges. d. Aerzte in Wien. 1862. Nr. 26 u. 27.

3) Prager Vierteljahresschrift. Bd. III. S. 109. 1863.

4) Recherches chimiques et physiologiques sur l'érytroxylon coca du Pérou et la cocaïn. Thèse de Paris 1868.

5) Citirt nach v. Anrep.

6) Archiv f. d. ges. Physiol. Bd XXI. S. 38. 1879.

7) Ber. d. XVI. Vers. d. ophth. Ges. zu Heidelberg 1884. S. 60. - Wiener med. Wochenschr. 1884. Nr. 43. S. 1276 u. Nr. 44. S. 1309.

8) Centralblatt f. prakt. Augenheilkunde. Bd. IX. S. 206. 1885. - Klin. Monatsblätter f. Augenheilkunde. Bd. XXIV. S. 169. 1886.

9) Im pharmakologischen Institute zu Strassbarg.

10) Centralbl. f. die med. Wissensch. 1885. Nr. 9. S. 146. 
Kritische u, experiment. Untersuchungen über die Irisbewegungen u. s. w. 111

Atropin noch mehr dilatirt wird. Umgekehrt nimmt auch die stärkste Atropinmydriasis nach Cocaineinträufelung noch zu (v. A n rep, Ad. Weber ${ }^{1}$, Schöler' ${ }^{2}$, Höltzke ${ }^{3}$, Jessop ${ }^{4}$, Bellarmi$\left.n \circ f^{5}\right)$ u. A.).

Wenn auch bei $\mathrm{Oculomotoriuslähmung} \mathrm{Cocain} \mathrm{die} \mathrm{Mydriasis}$ vermehrt ( $A d$. Weber, Bellarmin off), so kann doch aus dieser Thatsache auf keine Beziehung des Alkaloides zum Oculomotorius oder Sphincter pup. geschlossen werden, obschon die mittelweite und auf Licht nicht reagirende Pupille bei voliständiger Oculomotoriuslähmung durch Atropin ebenfalls noch etwas erweitert wird, wie zuerst $R u e^{6} \mathrm{e}^{6}$ ) gefunden hat. Auch nach Entfernung des Oculomotorius bei Thieren erhielten Bernard, Budge ${ }^{7}$ ) und Donders ${ }^{8}$ ) durch Atropin Zunahme der Pupillenweite. Nach Donders folgt hierans nicht, dass bei der Atropinwirkung am Auge der Sympathicus betheiligt ist. Er nimmt an, dass nach Unterbrechung der Oculomotoriusbahn das Ganglion ciliare noch einen Tonus ausïbe, der erst auf Atropin schwinde. Da Cocain die atropinisirte Pupille noch mebr erweitert, so behält es also nach Lähmung des Oculomotorius seine Wirkung noch bei. Hiermit stimmt überein, dass, wie ich gefunden habe, Durchtrennung dieses Nerven keinen wesentlichen Einfluss auf die weitere Vergrösserung der Pupille durch Cocain ausibbt.

Die Verschiedenheit von Cocain und Atropin geht besonders deutlich daraus hervor, dass die cocainisirte Pupille anf Belich. tung und Convergenz ausgiebig reagirt (Koller, Königstein ${ }^{9}$ ), A. Weber u. A.), während bei guter Atropinwirkung die Regenbogenhaut vollständig bewegungslos ist und gegen alle Reize, welche den Oculomotorius treffen, völlig unempfindlich bleibt; weder Reizung des Oculomotorius (Bernstein u. Dogiel ${ }^{10}$ ), Adam ük ${ }^{11}$ ) oder der Ciliarnerven (Hensen u. Völckers), noch der Endigungen durch Lichtreiz oder Muscarin bewirken Myose.

Da die Cocainpupille in direetem Sonnenlicht sich contrahirt, so

1) Klin. Monatsbl. f. Augenheilkunde 1884.

2) Berliner med. Gesellsch. v. 7. Jan. 1885, nach Centralblatt für praktische Augenheilkunde, Bd. IX. 1885. S. 28.

3) Ebenda; ferner klin. Monatsbl. f. Augenheilkunde 1887. S. 104.

4) The Practioner. 1885. S. 1.

5) Klin. Monatsbl. f. Augenheilkunde. Bd. XXIII. 1885. S. 511.

6) a. a. O. Bd.I. S. $101 . \quad$ 7) a. a. O. S. $182 . \quad$ 8) a. a. O. S. 498.

9) Wiener med. Presse 1884. Nr. 42. S. 1340. Nr. 43. S. 1365.

10) Verhandl. des naturw.-med. Vereins zu Heidelberg 1866. Bd. IV. S. 28. Centralbl. f. d. med. Wissensch. 1866. S. 453.

11) Centralbl. f. d. med. Wissensch. 1870. Nr. 12. S. 177. 
ist ihre Grösse abhängig von der Intensität der Beleuchtung. Es sind daher die Umstände zu berücksichtigen, unter denen die Beobachtung stattfindet. Es ist so verständlich, wenn Königistein und Höltzke angeben, dass der Unterschied in der Weite einer cocainisirten und einer normalen Pupille bei directem Lichteinfall geringer ist, als bei Beschattung der Augen (s. oben S. 98). Da im Dunkeln beide Pupillen weit sind, etwa wie es der aufgehobenen reflectorischen Oculomotoriusreizung entspricht, so darf als sicher angenommen werden, dass die cocainisirte Iris in vollständiger Dunkelheit einen schmäleren Saum bildet, als am nicht vergifteten Auge. Ob dagegen unter diesen Bedingungen die cocainisirte Pupille ebenso gross oder grösser ist, als die atropinisirte, lässt sich so nicht entscheiden, da Atropin die Pupille stärker erweitert, als dem Fortfall des von der Netzbaut ausgehenden Reflexes auf den Sphineter entspricht. Jedenfalls geben beide Alkaloide zusammen die bedeutendste Erweiterung, die grösser ist, als sie sich durch die Anwendung eines einzelnen beim Feblen jeder Beleuchtung erreichen lässt.

Das ungleiche Verhalten der mit Cocain oder Atropin vergifteten Papille zeigt, wie man feststellen kann, ob beim Menschen eine Mydriasis auf einer Lähmung des Oculomotorius und Sphincters, oder auf anderen Ursacben beruht. Im ersteren Falle ist die Pupille im Vergleich mit dem anderen normalen Auge bei starker Belenchtung verhältnissmässig weit. Bei schwacher Beleuchtung ist diese Differenz zwischen beiden Augen gering. Wenn dagegen die Mydriasis unabhängig vom Oculomotorius und Sphincter besteht, so wird umgekehrt beobachtet, dass mit Verminderung der Helligkeit die Pupillendifferenz immer grösser wird; bei sehr hellem Tageslicht kann sie sogar verschwinden.

Ein weiterer Unterschied der Cocain- von der Atropinwirkung am Auge ist darin begründet, dass die Cocainpupille durch Pilocarpin, welches die Endigungen des Oculomotorius reizt, leicht aufgehoben wird (Ad. Weber, Höltzke u. A.). Ich sah dasselbe vom Muscarin. Physostigmin, welches den Sphincter pup. selbst zur Contraction veranlasst, verengt ebenfalls leicht die mit Cocain vergiftete Iris. (Ad. Weber, Schweigger. ${ }^{1}$ )

b) Besondere Erscheinungen am cocainisirten Auge.

Die eigenartige Stellung des Cocains unter den auf das Auge wirkenden Giften tritt auch bervor, wenn wir das Gesammtbild der am Auge hervorgerufenen Erscheinungen berücksichtigen.

1) CentralbI. f. prakt. Augenheilkunde. Bd IX. 1885. S. 1. 
Kritische u. experiment. Untersuchungen über die Irisbewegungen u. s. w. 113

Regelmässig beobachtet wird eine Erweiterung der Lidspalte. Sie beruht zum Theil auf einer Hebung des Oberlides (Ad. Weber). Theilweise wird sie verursacht durch eine Protrusion des Bulbus. Letztere wurde experimentell an Fröschen (Moreno y Maïz), Hunden (Vulpian ${ }^{1}$ ), Durdufi ${ }^{2}$ ) und Kaninchen (Schöler, Durdufi) beobachtet. Königstein und Schenkl ${ }^{3}$ ) beschreiben sie auch beim Menschen.

Bei stärkerer localer Cocainisirung ist ferner der intra oculare Druck herabgesetzt (A. Weber, de Magri u. Denti ${ }^{4}$ ), Bellarminoff, Zieminski ${ }^{5}$ ), van Millingen 6 ) u. A.).

Ein besonders wichtiges Symptom der Cocainvergiftung ist die Gefässverengernng. Verschmälerung der peripheren Arterien wurde zuerst bei Warmblütern von Schroff und an der Zunge und Schwimmhaut der Frösche von v. Anrep beobachtet. Auf eine solche Gefässcontraction bezog man die Blässe und Trockenheit der Conjunctiva und die Herabsetzung der Temperatur des Conjunctivalsackes bei localer Application (Ad. Weber). de Magri u. Denti sahen das Kaliber der Netzhautgefässe abnehmen. Eversbusch i) führt auch die mydriatische Wirkung des Cocains auf diese Gefässveränderung zurück. Er beobachtete an der Iris albinotischer Kaninchen und junger Katzen eine so starke Verschmälerung der Irisgefässe, dass letztere schliesslich unsichtbar waren bis auf den ebenfalls verengerten Circulus arteriosus iridis major, von dem noch hier und da der Anfang eines grösseren Astes ausging. Diese Angaben werden von E. Pflüger u. Kronecker nicht bestätigt. Es ist aber möglich, dass in den Versuchen, welche die Letzteren anstellten, das Cocain nicht in genügender Menge zur Iris gedrungen war. Auf Verengerung der Irisgef ässe bezieht es Eversbusch ${ }^{8}$ ) ferner, dass nach Abfluss des Kammerwassers die cocainisirte Pupille sich nicht zasammenzieht, während unter denselben Verhältnissen die atropinisirte sich contrahirt. ${ }^{9}$ )

1) Compt. rend. T. XCIX. p. 836 et 885.1884.

2) Deutsche med. Wochenschr. Bd. XIII. Nr. 9. S. 172. 1887.

3) Wiener med. Presse. 1885. Nr. 1.

4) Citirt nach Jahresber. d. Ophthalm. von $\mathrm{Nagel-M}$ ichel. 1884. S. 317.

5) Exp. u. klin. Beiträge zur Frage über die Anwendung des Cocains in d. Ophthalmologie. Inaug--Diss. Dorpat 1884. - Klin. Monatsbl. f. Augenhk. Bd. XXXIII. 1885. S. 301.

6) Centralbl.f. prakt. Augenheilkunde. Bd. IX. 1885. S. 18.

7) Münchener med. Wochenschr. Bd. XXXII. 1885. Nr. 1. S.1. Nr. 2. S. 14. - Centralbl. f. prakt. Augenheilkunde. Bd. IX. 1885. S. 289.

8) Zeitschr. f. vgl. Augenhk. Bd. III. 1885. S. 40. A r chiv f. experiment. Pathol, a. Pharmakol. XXX. Bd.

9) Vgl. Budge a.a. O. S. 64 . 
Unter den Symptomen am Auge ist eine Erscheinung noch zu besprechen, welche zuerst darauf hinzudeuten schien, dass der Oculomotorius doch nicht vollständig unbetheiligt sei. Von zahlreichen Autoren, namentlich Koller, Königstein, Nettleship ${ }^{1}$ ), Weber, Jessop, Knapp, E. Pflüger, Landolt, Bellarminoff, Zieminski, wurde eine Lähmung der Accommodation beschrieben. Diese, in der Regel geringfügig, könne bisweilen einen höheren Grad erreichen. We b e r suchte die Ursache für die Betheiligung der Accommodation in einer partiellen Erregung des Ciliarmuskels. Nettleship und Eversbusch beschuldigen eine Ischämie dieses Muskels. In einer späteren Mittheilung vermuthet Eversbusch als Ursache eine oberflächliche Trübung der Hornhaut (Bunge ${ }^{2}$ ), Laqueur $\left.{ }^{3}\right)$ bei intactem Accommodationsapparat. Vorher hatte schon Höltzke eine jede Beschränkung der Accommodation auf das Entschiedenste in Abrede gestellt. Ihm schloss sich Schöler an, welcher fand, dass die Abrückung des Nahepunktes schwand, wenn die starke Mydriasis durch ein vorgehaltenes Diaphragma corrigirt wurde. Diese Angabe wird von E. Pflüger nicht bestätigt.

Aus Versuchen, welche ich zu anderen Zwecken vorgenommen habe, muss ich schliessen, dass allerdings nach Cocaineinträufelung in dem Bindehautsack Erscheinungen auftreten, welche auf Vorgänge im Accommodationsapparat zu beziehen sind. Ihr Charakter ist in mancher Beziehung eigenartig. Der Nahepunkt ist hinausgeriekt. Auch habe ich, wie A. Weber und E. Pfl üg er, die Veränderung des Fernpunktes bei Myopen wahrgenommen. Letzterer scheint herangeriickt; ich möchte eher sagen, auf einen bestimmten Accommodationsimpuls ist die Einstellung etwas zu stark. Die Veränderung beginnt am Fernpunkt und schreitet von hier aus nach dem Nahepunkt hin in verschieden grosser Ausdehnung; dass Fern- und Nahepunkt zusammenfielen, habe ich nicht beobachtet; ich fand stets eine nicht betheiligte Strecke, welche in einem Falle allerdings nur $3 \mathrm{~cm}$ lang war. Der Vorgang pflegt sehr passagerer Natur zu sein. Eigenthümlich erscheint dabei, dass er, wie Evers bu s ch zeigte, durch Gläserprüfung nicht nachgewiesen werde konnte. E. Pflïger constatirte bei einem Knaben mit vollständiger Accommodationslähmung eine leichte Zunahme der Hyperopie.

Für diese Veränderung des Accommodationsapparates, welche mehr als eine scheinbare ist, darf nicht an eine directe Einwirkung

1) Ophthalm. Society. 1884. 11. Dec.

2) Klin. Monatsbl. f. Augenheilkunde. Bd. XXIII. 1885. S. 402.

3) Bericht über den VII. intern. Ophth.-Congress zu Heidelberg 1888. S. 156. 
Kritische u. experiment. Untersuchungen über die Irisbewegungen u. s. w. 115

des Cocains auf den Oculomotorius oder Ciliarmuskel gedacht werden. Sie muss vielmebr auf die Veränder ung der Gefässe und wahrscheinlich hieraus resultirende Vorgänge bezogen werden. Wie durch Contraction der Irisgefässe Mydriasis entsteht, so werden auch in der ibrigen Uvea Bewegungen hervorgernfen. Es sind so Erscheinungen möglich, welche in der Gegend des Fernpunktes eine gewisse Aehnlichkeit mit einer Anspannung der Accommodation besitzen. Die Hinausrïckung des Nahepunktes scheint mehr auf einer Aenderung des intraocularen Druckes und der brechenden Medien') zu bertuhen. Ich erinnere daran, dass Petit und Cl. Bernard nach Sympathicusdurchschneidung eine Abflachung der Cornea beobachteten. $\left.{ }^{2}\right)$ Wichtig ist ferner der Befund Nagel's ${ }^{3}$ ), welcher angiebt, dass bei Lähmung des Sympathicus die Refraction des gleichseitigen Auges zunimmt.

c) Ursache der Pupillenerweiterung durch Cocain.

Die angeführten Symptome der Cocainvergiftung am Auge haben die grösste Aehnlichkeit oder stehen in directem Gegensatz mit den Erscheinungen, welche als Folge einer Reizung oder Durchschneidung: des Halssympathicus (Petit, Biffi, Cl. Bernard u. A.) bekannt sind oder bei Zuständen zur Beobachtung gelangen, welche auf eine Erkrankung desselben bezogen werden. Man glaubte daher annehmen zu müssen, dass Cocain die Endigungen des Sympathicus im Auge reize (A. Weber u. A.). Für die Richtigkeit dieser Anschanung sprechen auch die Beobachtungen an Personen mit Augenaffectionen, die auf einer Störung des Sympathicus beruhten. Reichenhe i m ${ }^{4}$ ) beschreibt einen Fall von angeborener Ptosis, in dem Cocain entschieden weniger kräftig wirkte. E. Pflüger untersuchte Leute mit Ptosis and Myosis infolge einer Lähmung des Sympathicus. Bei denselben versagte Cocain vollständig. Ich hatte Gelegenheit, in der Strassburger Klinik mehrere Fälle der Art ${ }^{5}$ ) zu sehen. Nach der localen Anwendung des Cocains war sowohl die Erweiterung der Pupille, als die Hebung des Oberlides sicher nachweisbar. Auf der gesunden war der Erfolg des Giftes allerdings grösser.

1) Vgl. Boss, S., Ueber die Veränderungen des Hornhautradius unter dem Einflusse von Atropin, Homatropin, Physostigmin und Cocain. Inaug.-Diss. Strassburg 1890.

2) Vgl. Leber a. a. O. S. 377.

3) Klin. Monatsbl. f. Augenheilkunde. 1873. S. 391.

4) Ebenda. Bd. XXII. 1884. S. 462.

5) Dieselben wurden inzwischen veröffentlicht. Hoffmann, P., Ueber den Horner'schen Symptomencomplex. Inaug.-Diss. Strassburg 1891. 
Die Untersuchung dieser Frage mittelst Durchschneidung des Halssympathicus wurde zuerst von Nikolsky ) vorgenommen. Er fand nach dieser Operation Cocain noch sehr wirksam am Auge. Auch die durch das Alkaloid herbeigefthrte Mydriasis schwand nicht bei der Nervendurchschneidung. Zieminski zieht den Schluss, dass Cocain den Sympathicus nicht antaste, sondern den Oculomotorins zu afficiren scheine. Höltzke gelangte zu der entgegengesetzten Ansicht. Nach der Sympathicusdurchschneidung wurde die Pupille durch Cocain weniger stark erweitert, als vorher; hieraus wird geschlossen, dass eine Betheiligung des Sympathicus vorliege. Bei Kaninchen machte ich dieselbe Beobachtung. Ich muss sie aber, soweit es diese Thiere betrifft, in anderer Weise deuten, denn ich sah, wie auch schon Berthold, dass nach elektrischer Reizung des peripheren Endes des durchschnittenen Halssympathicus an der cocainisirten Pupille keine Verstärkung der Erweiterung eintrat. Letztere hätte erfolgen muissen, wenn die Enden des Sympathieus in der Iris sich nicht schon im Zustande der stärksten Erregung befunden hätten. Der Fortfall eines centralen Tonus des Sympathicus kann nicht die Ursache der verminderten Mydriasis sein. Ieh möchte vermuthen, dass im Auge selbst gelegene Veränderungen complicirterer Natur anzunehmen sind. Ich meine die Beziehung des Sympathicus zu m intraocularen Druck. Diese ist aber noch nicht hinreichend anfgeklärt. Eine Herabsetzung des Druckes würde die geringe Myose nach Sympathicusdurchschneidung vollständig erklären. Ich befinde mich in Uebereinstimmung mit $\mathrm{Nagel}$, welcher bereits auf den $\mathrm{Zu}$ sammenhang von Myose und Druckverminderung hinwies.

Ich darf hier wohl die zuerst von Biffi gemachte Beobachtung anführen, dass nach Sympathicusdurchschneidung auch die atropinisirte Pupille weniger weit ist. $\mathrm{Braun}^{2}$ ) gab die Erklärung, dass durch die Zerstörung des Sympathicus am Halse die Innervation durch denselben versehwunden sei. Um diesen Betrag sei die Atropinpupille enger, indem der zum „Dilatator" gehende Tonus fortfällt. Auch ich fand bei stärkster Atropinisirung die Differenz unverändert. Ich halte es für wahrscheinlich, dass die eigentliche Ursache nicht in dem Centralnervensystem, sondern im Auge selbst zu suchen ist, vielleicht in der oben angegebenen Weise.

Sichergestellt wurde die Bedentung des Sympathieus durch Schöler, welcher fand, dass 24-36 Stunden nach der Exstirpation

1) Citirt nach Zieminski.

2) Archiv f. Ophthalm. Bd, V (2). S. 112. 1859. 
Kritische u. experiment. Untersuchungen über die Irisbewegungen u. s. w. 117

des Ganglion cervicale supr. Cocain die Pupille nicht mehr erweitert. E. Pfl ü ger erhielt dasselbe Resultat.

Wenn man bei Kaninchen in der bekannten Weise das Ganglion cerv. supr. exstirpirt, so ist Cocain zunächst noch gut wirksam. Es gelang mir aber ebensowenig wie Höltzke, die Pupille maximal zu erweitern. Der Unterschied hatte, wie schon erwähnt, bei Kaninchen die Grösse der sogenannten centralen Dilatatorinnervation. Meine Ansicht iber letztere habe ich vorhin gesagt. Während im Allgemeinen ein paar Tage nach der Sympathicuszerstörung die Iris auf Cocain überhaupt nicht mehr reagirt, so giebt es doch Fälle, in denen die Empfänglichkeit desselben weniger schnell nachlässt. Wurde das Auge von der Operation an einer starken Cocainwirkung ausgesetzt, so hielt letztere viel länger an. Es dilatirte das Alkaloid bei einem Thiere noch am 5. Tage die Pupille um $1,0 \mathrm{~mm}$ und am 8. Tage um $0,5 \mathrm{~mm}$. Der Betrag ist vielleicht noch grösser, da der Einfluss des Alkaloides über Nacht möglicher Weise nicht völlig geschwunden war.

Das schnelle Nachlassen der Erregbarkeit der Nervenenden nach Entfernung des Ganglion cervic. supr. kann also durch Cocain aufgehalten werden. Aber auch wenn die Erregbarkeit vollständig erloschen erschien, liess sie sich in geeigneter Weise wieder anfachen, so dass unter geeigneten Umständen noch erheblich später, als angegeben, Pupillendilatation erhalten wurde. Wenn bei einem Kaninchen einige Tage nach der Exstirpation des obersten Halsganglions die Degeneration des Sympathicus so weit fortgeschritten war, dass Cocain die Pupille nicht mebr erweiterte, so trat diese Wirkung wieder ein, nachdem vor der Einträufelung des Alkaloides die Hornhautvermittelst Elektricität direct gereiztwordenwar. An einem solchen Auge entfaltete Cocain wieder seine Kraft. Die Mydriasis konnte selbst annähernd maximal, demnach grösser als unmittelbar nach der Sympathicuszerstörung sein. Die Erklärung hiervon lässt sich in folgender Weise geben. Durch die vorhergehende Elektrisirung wurden sämmtliche Iriselemente stark gereizt; sie befanden sich infolgedessen in einem Zustande erhöhter Erregbarkeit. Cocain war wieder wirksam, und der von ihm ausgeübte Reiz genügte, um dem dilatirenden Apparat in der Iris das Uebergewicht zu geben. Es kommt aber noch ein anderer Umstand in Betracht. Noch 13 Tage nach der Sympathicusverödung erhielt ich diese Dilatation der Pupille an einem Kaninchen, welches 5 Tage vorher zum letzten Male cocainisirt worden war. Unmittelbar nach der Sympathicusdurchschneidung betrug die Cocainmydriasis $9 \mathrm{~mm}(2 \mathrm{~mm}$ weniger als 
auf der anderen Seite). 13 Tage später trat nach voraufgegangener elektrischer Bulbusreizung durch Cocain eine Pupillenerweiterung von $10,5 \mathrm{~mm}$ ein; das andere Auge, welches nur mit Cocain vergiftet wurde, zeigte $11,0 \mathrm{~mm}$ Dilatation. Ein Unterschied in der Grösse beider Pupillen war also kaum noch vorhanden. Dieses Resultat lässt sich nicht ausschliesslich dureh den Irissympathicus erklären. Dass die Mydriasis hauptsächlich auf letzteren zu beziehen ist, kann nicht zweifelhaft sein, denn die Verödung des Nerven am Halse hatte Cocain unwirksam gemacht. Der Versuch ist verständlich, wenn man annimmt, dass durch die elektrisehe Reizung neben der Aenderung der Erregbarkeit der Iriselemente auch die nach Durchschneidung des Halssympathicus eintretende Pupillenverengerung beeinflusst wird. Falls die Myose anf einer Herabsetzung des intraocularen Druckes beruht, so darf angenommen werden, dass durch die elektrische Hornhautreizung reflectorisch die Druckverminderung nahezu ausgeglichen wurde. Ein solcher Vorgang ist wenigstens möglich. ${ }^{1}$ )

Aus den angefuibrten Thatsachen muss geschlossen werden, dass Cocain am Auge die Endigungen des Sympathicas reizt. Für eine Mitbetheiligung des Oculomotorius lassen sich, wie gezeigt wurde, keine stichhaltigen Grïnde anfübren.

\section{d) Einfluss des Trigeminus auf die Pupille des cocainisirten Auges.}

Ein besonderes Interesse bieten die Beziehungen zum Trigeminus, da die Hornhautanästhesie durch Cocain auf eine Lähmung der sensiblen Fasern dieses Nerven zurïckgefithrt wird.

Versuch: Mittelgrosses Kaninchen. 1,5 eg Morpl. muriat. subcutan. $7 \mathrm{~h} .5 \mathrm{~m}$. Trigeminusdurchschneidung auf der linken Seite. Auftreten der charakteristischen Erscheinungen:

7 h. $10 \mathrm{~m}$. Pupillenweite L. 2,5 R. $8,0 \mathrm{~mm}$

$7 \mathrm{~h} .15 \mathrm{~m} . \quad=\quad=5,0=9,0=$

$7 \mathrm{h.} 20 \mathrm{~m} . \quad=\quad=8,5=10,0=$ muriat. in beide

$\begin{array}{ll}7 \mathrm{~h} .25 \mathrm{~m} . & =10,0=10,0= \\ 7 \mathrm{~h} .30 \mathrm{~m} . & =\end{array} \quad$ Augen.

Wie dieser Versuch zeigt, wirkt Cocain sehr prompt an einem Auge, dessen Trigeminus durchschnitten ist. Die Wirksamkeit des Alkaloides erscheint eher gesteigert, denn nur unerheblich später als auf dem anderen, sonst normalen Auge wird die Pupille maximal erweitert, obschon sie unmittelbar nach der Nervendurchschneidung:

1) Vgl. Leber a.a. 0. S. 377. 
stark verengt ist. Die Reaction der durch Cocain dilatirten Pupille auf Belichtung ist durch die Trigeminusausschaltung natiirlich nicht vernichtet. Die schliesslich gleichmässige Erweiterung beider Pupillen muss darauf bezogen werden, dass der intraocalare Druck durch die Trigeminusdurchschneidung nicht nennenswerth alterirt $\mathfrak{w r d e}^{1}$ ), oder es ist auch nicht richtig, dass die Myose nach Durchschneidung des Halssympathicus auf einer Hypotonie des Bulbus beruht.

Mit dem eben besprochenen Versuche vergleiche man den folgenden :

Nach der Trigeminusdurchschneidung bei einem kleinen Kaninchen beträgt der Durchmesser der Pupille 2,0 $\mathrm{mm}$, die andere ist $6,0 \mathrm{~mm}$ breit. In $Z$ wisehenräumen von 5 Minuten wird in beide Augen Atropinsulfat in 1 proc. Lösung eingeträufelt. 30 Minuten später nach 5 maliger Einträufelung beträgt die Pupille 4,0 und 8,0 mm. Auch am folgenden Tage bleibt noch eine Differenz von $2,0 \mathrm{~mm}(7,0$ and 9,0 mm Durchmesser), welche durch weitere Atropinanwendung nicht ausgeglichen wird. Am 3. Tage ist noch ein Unterschied von 1,0 mm vorhanden. Erst am 4. Tage waren beide Pupillen gleichweit.

Am 1. Tage erweiterte also Atropin beide Pupillen um dieselbe Grösse. Die Differenz bei Beginn der Vergiftung blieb erhalten. Atropin muss demnach unabhängig von den Momenten wirken, welche bei der intracraniellen Durchschneidung des Trigeminus die Myose herbeifuhren. Es erweitert nämlich beide Pupillen um die Grösse, welche auf der Ausschaltung des Oculomotorius beruht. Dasselbe Verhalten findet man bekanntlich nach der Exstirpation des Ganglion cervic. supr. Träufelt man bei einem auf diese Weise operirten Kaninchen Atropin in beide Augen, so dilatiren sich ebenfalls beide Pupillen um dieselbe Grösse. Da Atropin in keiner Beziehung zum Sympathicus steht, so bleibt die auf der einseitigen Ausschaltung dieses Nerven beruhende Pupillendifferenz erhalten.

Der Gegensatz, welcher auch an Thieren mit intracranieller Trigeminusdurchschneidung zwischen beiden Alkaloiden besteht, ist bemerkenswerth. Es ist dies kaum durch die Thätigkeit eines rom Sympathicus innervirten „M. dilatator pupillae" zu erklären. Man könnte nicht verstehen, dass die aus jenem Eingriff resultirende grosse Pupillendifferenz durch Cocain in so kurzer Zeit und vollständig ausgeglichen wurde. Ein, wenn auch noch so kleiner Unterschied in der Weite beider Pupillen müsste doch wobl zurückbleiben, zumal da

1) Vgl. Leber a. a. 0. S. 379. 
die pupillenverengernde Kraft der Trigeminusdurchschneidung ziemlich bedeutend ist. Ich habe daher, wie bereits Gruenhagen, gefunden, dass durch diese Operation die Atropinmydriasis vollständig oder vielmehr nahezu vollständig aufgehoben wird (Weite der Pupille $3,5 \mathrm{~mm}^{1}$ ).

Ein Einfluss des Cocains auf den Trigeminus ist unleugbar. Zur Erklärung desselben können wir zwischen folgenden Möglichkeiten wählen. Entweder afficirt Cocain diejenigen Trigeminusfasern, welchen die Verengerung der Pupille nach der Durchschneidung des Nerven zugeschrieben werden muss ${ }^{2}$ ), oder es steht in einer gewissen Beziehung zu dem Angriffspunkte derselben Fasern in der Iris. Auf eine Abhängigkeit des Trigeminus und Sympathicus ron einander lassen schon die Untersuchungen von Donders und Brondgeest ${ }^{3}$ ) schliessen, welche beobachteten, dass nach Trigeminusdurchschneidung Reizung des Sympathicus unter 11 Fällen nur 7 mal mit Erweiterung der Pupille antwortete. $W$ egner ${ }^{4}$ ) erhielt ein ähnliches Resultat.

Da nach Trigeminusdurchschneidung im weiteren Verlaufe leicht Hornhautulcerationen auftreten, so können secundäre Veränderungen in der Regenbogenhaut ${ }^{5}$ ) sich einstellen, welche der Cocainmydriasis entgegenkämpfen. Es beruht die Aenderung nicht darauf, dass der Angriffspunkt des Alkaloides Umwandlungen erleidet, welche mit den Vorgängen an den Endigungen des Sympathicus nach der Exstirpation des obersten Halsganglions in Parallele zu setzen wären.

Bei einem keinen Kaninchen war es bereits 3 Tage nach der intracraniellen Trigeminusdurchschneidang zu einem Hornhautgeschwür gekommen. An diesem Auge erweiterte Cocain die Pupille anf 7,5 mm, während auf der anderen Seite $9,0 \mathrm{~mm}$ gemessen wurden. Vor der Vergiftung hatte die Differenz 1,0 $\mathrm{mm}$ betragen. Am folgenden Tage war die Hornhaut perforirt. In der vorderen Kammer fand sich ein niedriges Hypopyon. Letzteres nahm am 5. Tage 1/4 der wiederhergestellten Kammer ein. Die beiderseits $4,0 \mathrm{~mm}$ weite Pupille wurde durch Cocain ziemlich gleichmässig dilatirt zu fast maximaler Mydriasis von $9,0 \mathrm{~mm}$ auf der operirten und von $9,5 \mathrm{~mm}$ auf der

1) Wenn erst der Oculomotorius und hierauf der Trigeminus durchtrennt wird, so entsteht Pupillenverengerung. Vgl. Budge a. a. 0. S.99.

2) Vgl. Leber a, a. 0. S. 364 .

3) Donders a. a. O. S. 491.

4) a. a. O. S. 10.

5) Siehe Leber, Th., Die Entstehung der Entzündung und die Wirknng der entzündungerregenden Schädlichkeiten u. s. w. Leipzig 1891. S. 478. 
Kritische u. experiment. Untersuchungen über die Irisbewegungen u. s. w. 121

normalen Seite. 3 Tage später war das Hypopyon, obschon das Auge nur mit Wasser gereinigt wurde, verschwunden und das Gesehwür in der Heilung begriffen. In diesem Versuche ist die verminderte Wirksamkeit des Cocains wohl nur zum Theil auf eine Alterirung der Resorptionsbedingungen zu schieben. Nach der Perforation des Geschwiires war das Alkaloid jedenfalls wieder gut wirksam. Eine Veränderung der Iris, welche ihre Beweglichkeit verminderte, ist daneben wahrscheinlich.

Wenn bei einem Kaninchen 7 Tage nach der intracraniellen Trigeminusdurchschneidung - es bestand bereits ein Hornhautgeschwür bei gleicher Pupillenweite beider Augen - das obere Halsganglion entfernt wurde, so kam bei der Einträufelung von Cocain in den Bindehautsack nur die letztere Operation in der früher beschriebenen Weise in Betracht. Unmittelbar nach dem Eingriff entstand daher durch Cocain keine vollständig maximale Mydriasis.

Nach diesen Versuchen muss ich annehmen, dass der Trigeminus nicht selbst afficirt wird. Seine Bedeutung muss daher so aufgefasst werden, dass der Apparat, durch welchen Cocain die Pupille dilatirt, von Trigeminus und Sympathicus zugleich innervirt wird. In dieser Beziehung wird man beide Nerven als Antagonisten ansehen können. Aus der verschiedenen Wirkung von Atropin und Cocain auf die Pupille nach Trigeminusdurchschneidung und unter Berücksichtigung der früher angeführten Punkte ist die weitere Folgerung wohl gerechtfertigt, dass der Dilatator pupillae in näheren Beziehungen zu den Gefässen der Regenbogenhaut steht. Am einfachsten wird man sich das in der Weise denken, dass der Dilatator einen Theil der Gefässwand selbst bildet. Die Annahme eines selbständigen und isolirten Muskels mit dieser Function ist auch für die Erklärung der Cocainwirkung am Auge überflüssig, erschwert vielleicht sogar das Verständniss.

Meine Versuche gestatten Schlüsse iiber die Zweckmässigkeit der Cocainbehandlung bei der Iritis. Wenn auch die Cocainmydriasis beim Menschen wenig bedeutend erscheint, so wurde oben besprochen, dass dies oft nur davon herrübrt, dass man bei sehr heller Beleuchtung untersuchte. Es wurde erwähnt, dass die Atropinpupille durch Cocain noch mehr erweitert wird. Die Myose bei Entztundung der Iris und nach Trigeminusdurchschneidung dïrfen zwar nicht gleich gesetzt werden, doch kann mit Cocain wegen seiner Einwirkung auf die Gefässe die Hyperämie der Iris bekämpft werden, dagegen wohl nicht direct die Infiltration. Hyperämie und Infiltration der Gewebe 
stehen aber in einem gewissen Zusammenhang. ${ }^{1}$ ) Es lässt sich annehmen, dass zumal im Beginn einer Iritis und in den späteren Stadien der Entzündung, wenn die Infiltration nachlässt, die Anwendung von Cocain neben Atropin zweekmässig ist, wie auch schon A. Weber, Knapp, Seböler u. A. hervorheben.

e) Einfluss des Cocains auf die Erscheinungen bei der directen Irisreizung.

Da Cocain die Pupille erweiternde Nerven reizt und die Sensibilität lähmt, so war zu erwarten, dass es die Erscheinungen bei der sogenannten directen Irisreizung modificirt. Bei diesem Verfahren werden ausser den museulösen und nervösen Elementen der Iris auch die sensiblen Nerven der Hornhaut und der vordere Theil der Retina afficirt. Reflectorische Vorgänge erschweren daher die Deutung.

Wenn der elektrische Strom die Retina trifft, so ist der Effect vielleicht derselbe wie bei der Netzhautreizung dureh Licht. Die Contraction des Sphincters erfolgt bei Kaninchen natürlich einseitig. Anzuführen ist hier die oben beschriebene kurzdauernde Erweiterung, welche bei Reizung der Hornhaut normaler Augen entsteht und auf eine Hemmung des von der Netzhaut ausgehenden Reflexes auf den Sphincter bezogen wurde. Diese Dilatation erschwert es sehr, die Bedeutung des Sympathicus festzustellen.

Am lebenden Thiere ist die Rolle, welche der directen Reizung der Iris selbst zukommt, nicht ganz leicht zu beurtheilen. Da der innere, den M. sphincter pup. bergende Theil der Regenbogenhaut dem elektrischen Strom nur schwer zugänglich ist, so muss letzterer oft stärker sein, als es sonst bei physiologischen Untersuchungen zweckmässig ist. Auf eine directe Betheiligung der Iris ist zunächst daraus zu schliessen, dass eine bestimmte Versuchsanordnung Verengerung oder Erweiterung der Pupille ergiebt. Letztere wird nach $\mathrm{Budge}^{2}$ ) am leichtesten beobachtet, wenn die Elektroden am Hornhautrand angesetzt werden. Umgekehrt ist es nach dem Vorgang von Bernstein und Dogiel's) zur Erzielung von Myose am besten, wenn man vier Elektroden in der Weise dem inneren Irisrand entsprechend anbringt, dass die diagonal gegenüberstehenden mit demselben Pol der secundären Rolle verbunden sind. Am isolirten Kopf und am frisch heraus-

1) Vgl. v. Reckling ha usen, Handbuch der allgem. Pathologie des Kreislaufs und der Ernährung. S. 25 u. 209. 1883.

2) a. a. O. S. 87 u. 88 .

3) Zeitschr. f. rat. Med. 3. Reihe. Bd. XXIX. S. 35. 1867. 
Kritische u. experiment. Untersuchungen über die Irisbewegungen a. s. w. 123

geschnittenen Auge treten diese Bewegungen am regelmässigsten ein, offenbar wegen der Ausschaltung des Centralnervensystems. Die Pupillenerweiterung bleibt nach Budge und Waller'), Gruenhagen und Hurwitz aus, wenn mebrere Tage vorher das oberste Halsganglion entfernt worden ist, selbst nach Anwendung von Atropin (Gruenhagen, Bessau). Aus der Angabe Kölliker's, dass nach Abtragung des inneren Theiles der Iris mit dem Sphineter bei Reizang Mydriasis entsteht, geht hervor, dass die Ciliarportion der Iris sich unabhängig vom Sphincter pup. verküizen kann. Hurwitz, welcher diese Versucbe wiederholte, fand, dass die Verkürzung der Iris bei Kaninchen ausblieb, wenn das Ganglion cervic, wenigstens 5 mal 24 Stunden vorher exstirpirt worden war, dass dagegen bei Katzen auch dann noch auf hinreichend starke Inductionsströme etwas Contractilität sich zeigte (vgl. S. 117). Ein ungleiches Verhalten beider Irisregionen Temperatureinflüssen gegenüber constatirten Gru e nhagen und Samkowy.2)

Am lebenden Thiere sind rom Trigeminus ausgehende Reflexe von grosser Bedeutung, Die starke Reizung sensibler Nerven durch den elektrischen Strom veranlasste mich häufig, den Kaninchen Morphin zu injiciren. Der Schmerz konnte sonst so gross sein, dass die Thiere laut aufschrieen, fast wie bei der intracraniellen Durchschneidung des fünften Gehirnnerven. Vom Trigeminus aus scheinen verschiedene Reflexe auf die Iris möglich zu sein. Bewiesen ist zunächst ein Ueberspringen auf diejenigen Trigeminusfasern, deren Reizung nach Cl. Bernard, Gruenhagen u. A. die Pupille bei Kaninchen verengt. Durch Experimente von Fowler ${ }^{3}$ ) und in neuerer Zeit von Gruenbagen, Rogow ${ }^{4}$ ) u. A. steht fest, dass bei isolirter Hornhautreizung am atropinirten Thiere Myose zu Stande kommt, welche sich natürlich nicht auf eine Contraction des Sphineters beziehen lässt. Von den Trigeminusendigungen aus ist aber auch Erweiterung der Pupille infolge der elektrischen Hornhautreizung möglich. Es kommt zunächst zu einer Steigerung des intraocularen Druckes und hierdurch zur Mydriasis. Auf diese Weise glaubte ich erklären zu müssen, dass die Pupillenverengerung bei Kaninehen mit Sympatbicusdegeneration zurïckgehen kann, wenn die Cornea von starken Strömen getroffen wurde.

Fassen wir Alles zusammen, so ist ersichtlich, dass bei der

1) Budge a. a. 0. S, 124.

2) Archiv f. die ges. Physiol. Bd. X. S. 165. 1875.

3) Siehe Budge a. a. 0. S. 87. Anm. 1.

4) Zeitschr. f. rat. Med. Bd. XXIX. 1867. 
sogenannten elektrischen Irisreizung verschiedenartige nervöse und musculöse Elemente erregt werden. Es würde daher zweckmässiger sein, jene Bezeichnung zu vermeiden.

Wird vor der Reizung Cocain an das Auge gebracht, so machen sich zunächst jene Uebelstände, welche aus der Unruhe des Thieres hervorgehen, nicht mehr bemerkbar, indem Aeusserungen von Schmerz nicht beobachtet werden. Dann ist selbst ein sehr starker Strom nicht mehr im Stande, Pupillenverengerung hervorzurufen. Das Ausbleiben der Myose muss theilweise auf die Reizung von die Pupille erweiternden Elementen durch Cocain bezogen werden. Hierzu kommt noch, dass wegen der Beziehungen, welche für den Dilatator zu den Irisgefässen anzunehmen sind, und wegen der Lähmung der Endigungen der sensiblen Trigeminusfasern von letzteren ausgehende Reflexe unwirksam sind.

Vergleichen wir hiermit das Verhalten des atropinisirten Auges. Es ist bekannt, dass an ihm die Myose nach directer elektrischer Reizung. sehr häufig ausbleibt. Man folgerte hieraus, dass Atropin die glatten Muskelfasern selbst weniger erregbar mache. ${ }^{1}$ ) Diese Thatsache liesse sich aber auch durch die Annahme erklären, dass der Sphincter pup. nach Lähmung des Oculomotorius anders, weniger reagirt, als der mit functionsfäbigen Nerven versehene. Von einer reflectorischen Erregung des Muskels von der Hornhant aus, welche Joh. Müller annahm, darf man vielleicht absehen.

Als weiterer Beweis, dass bei der elektrischen Hornhantreizung am lebenden Kaninchen Reflexe eine bedeutende Rolle spielen, ist anzuführen, dass auch am $\mathrm{zw}$ eite $\mathrm{n}$ Auge Bewegungserscheinungen von Seiten der Iris vorkommen. Ich kann die Angaben von $\mathrm{Hur}$ witz, Gruenhagen und Jesner ${ }^{1}$ ) bestätigen, dass auf Reizung del Hornhaut eines Auges beiderseits die Pupille sich rerengt. Ich habe am zweiten Auge eine Myose gesehen, welche die am ersten elektrisch gereizten uibertraf. Jene konnte fast maximal sein (bis zu $3,0 \mathrm{~mm}$ ). Am cocainisirten Auge habe ich sie nicht gesehen, indem die "Dilatator"-Reizung durch das Alkaloid offenbar nicht überwunden wurde, sei es dass die verengernde Kraft zu gering war, sei es dass Sympathicus und Trigeminus hinsichtlich der Gefässwirkung am Kaninchenauge Antagonisten sind. Vom cocainisirten Auge aus habe ich dagegen ganz unbedeutende Bewegungen der zweiten Iris erhalten. Ich sah an dieser eine geringfügige Zusammenziehung und Wiederausdehnung der Pupille. Durch Cocain waren die Endigungen der

1) Vgl. Rossbach u. Fröhlich, Verh. d. physib.-med. Ges. in Würzburg. Neue Folge. Bd. V. S. 1. 1873. 
Kritische $u$. experiment. Untersuchungen über die Irisbewegungen u. s. w. 125

sensiblen Nerven gelähmt, der Stamm selbst und die Endigungen in der Iris blieben aber dem elektrischen Reize noch zugänglich. Dass neben den sensiblen Nerven der Hornhant auch diejenigen der Iris den Reflex vermitteln, geht aus den Versuchen von Mooren

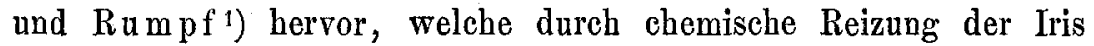
nach Entfernung der Hornhant ausgesprochene Gefässreflexe am zweiten Auge erhielten.

Ausser den Versuchen mit Cocain spricht auch das Resultat, welches ich bei Thieren mit Trigeminusdurchschneidung erhalten habe, dafur, dass dieser Nerv bei dem Vorgange betheiligt ist. An in dieser Weise einseitig operirten Thieren fehlte die reflectorische Myose am zweiten Auge und zwar nach beiden Richtungen hin. Es ist also anzunehmen, dass dieser Reflex dureh die sensiblen und vasomotorischen Trigeminusfasern vermittelt wir d.

Die zuletzt mitgetheilten Thatsachen lassen sich für die Erklärang der sympathischen Neurose (Leber) verwerthen, denn auch bei dieser wird ein Reizzustand auf das zweite Auge reflectorisch iibertragen. Inwieweit dabei ausschliesslich die Trigemini und ausserdem noch andere Nerven zu berücksichtigen sind, kann natürlieh an Kaninchen wegen der grossen Selbständigkeit beider Augen dieser Thierklasse nicht festgestellt werden.

1) Centralbl. f. d. med. Wiss. 1880 . Nr. 19. 